\title{
Dynamical formation of a hairy black hole in a cavity from the decay of unstable solitons
}

\author{
Nicolas Sanchis-Gual, ${ }^{1}$ Juan Carlos Degollado, ${ }^{2}$ José A. Font, ${ }^{1,3}$ Carlos Herdeiro, ${ }^{4}$ and Eugen Radu ${ }^{4}$ \\ ${ }^{1}$ Departamento de Astronomía y Astrofísica, Universitat de València, \\ Dr. Moliner 50, 46100, Burjassot (València), Spain \\ 2 Instituto de Ciencias Físicas, Universidad Nacional Autónoma de México, \\ Apdo. Postal 48-3, 62251, Cuernavaca, Morelos, México. \\ ${ }^{3}$ Observatori Astronòmic, Universitat de València, \\ C/ Catedrático José Beltrán 2, 46980, Paterna (València), Spain \\ ${ }^{4}$ Departamento de Física da Universidade de Aveiro and Centre for Research and Development \\ in Mathematics and Applications (CIDMA), Campus de Santiago, 3810-183 Aveiro, Portugal
}

(Dated: November 2016)

Recent numerical relativity simulations within the Einstein-Maxwell-(charged-)Klein-Gordon (EMcKG) system have shown that the non-linear evolution of a superradiantly unstable ReissnerNordström black hole (BH) enclosed in a cavity, leads to the formation of a $\mathrm{BH}$ with scalar hair. Perturbative evidence for the stability of such hairy BHs has been independently established, confirming they are the true endpoints of the superradiant instability. The same EMcKG system admits also charged scalar soliton-type solutions, which can be either stable or unstable. Using numerical relativity techniques, we provide evidence that the time evolution of some of these unstable solitons leads, again, to the formation of a hairy BH. In some other cases, unstable solitons evolve into a (bald) Reissner-Nordström BH. These results establish that the system admits two distinct channels to form hairy BHs at the threshold of superradiance: growing hair from an unstable (bald) $\mathrm{BH}$, or growing a horizon from an unstable (horizonless) soliton. Some parallelism with the case of asymptotically flat boson stars and Kerr BHs with scalar hair is drawn.

PACS numbers: 95.30.Sf, 04.70.Bw, 04.40.Nr, 04.25.dg

\section{INTRODUCTION}

In two recent papers [1, 2, we have investigated the non-linear evolution of the superradiant instability of a Reissner-Nordström (RN) black hole (BH), triggered by a charged scalar field confined to a cavity, which surrounds the $\mathrm{BH}$. This system is not only afflicted by superradiant instabilities - unlike asymptotically flat $\mathrm{RN}$ BHs [3, 4] - but also allows a sufficiently fast development of the instability [5], and hence a fully non-linear numerical treatment of the evolution. Moreover, within this system, superradiant instabilities occur even within spherical symmetry, making their treatment simpler.

Using numerical relativity techniques, we evolved in 1, 2 the Einstein-Maxwell-(charged-)Klein-Gordon (EMcKG) system, and showed that the endpoint of the instability is a hairy $\mathrm{BH}$ of the sort first discussed in 9 (see also [10]). In the appropriate gauge, the scalar hair is a stationary state characterized by a real frequency that equals the threshold frequency for the superradiant instability. Thus, these BHs exist at the threshold of superradiance, making them charged analogues of the (rotating) Kerr BHs with scalar [11, 12] and Proca hair [13. discovered in asymptotically flat spacetime (see also [1416. for generalizations with charge and self-interactions and [17 19] for analogue solutions in higher dimensions).

Besides the hairy BHs, the same EMcKG system allows for soliton-like solutions, which are everywhere regular and horizonless [20, 21. Indeed, the hairy BHs may be regarded as a bound state of these solitons with a horizon, in the same way that Kerr BHs with scalar (or Proca) hair can be regarded as a bound state of rotating boson stars [22] (or rotating Proca stars $[23]^{1}$ ) with a horizon (see, e.g. [26, 27], for discussions of horizons inside solitons). In [20, 21] the linear stability of the EMcKG solitons in a cavity was recently addressed and it was shown that some of these solutions are unstable against linear spherical perturbations. A natural question to ask is what is the end-point of the instability and, in particular, if the decay of these unstable solitons could yield a different channel for the formation of the aforementioned hairy BHs.

In this paper we shall address these questions, by using similar numerical methods to the ones used in [1, 2]. As we shall discuss below, there are different possible schemes to consider when evolving these EMcKG solitons. The reason is that the "cavity", which is something virtual for the stationary soliton solutions, needs to be made concrete, and imposes a concrete rule in the dynamical evolution of the system. Different schemes result from a different choice of rule, and ultimately, they reflect different dynamical problems. We shall illustrate this explicitly, by considering two different schemes. For these two possibilities, albeit we observe some quantitative and

\footnotetext{
${ }^{1}$ Static Proca stars have been recently generalized to include charge 24 or a cosmological constant 25].
} 
qualitative differences, there is a universal feature: we shall provide numerical evidence that indeed a hairy $\mathrm{BH}$ at the threshold of superradiance may form from the decay of an unstable soliton. But we also observe, for both schemes, that some unstable solitons decay into a (bald) $\mathrm{RN} \mathrm{BH}$, rather than a hairy one.

This paper is organized as follows. We start in section III by briefly describing the stationary solitonic solutions of the EMcKG system in a cavity [20, 21, which we have reproduced, and that will provide us with the initial data for our numerical evolutions. The framework for these evolutions is briefly reviewed in section [II] wherein both schemes are discussed and the corresponding numerical results are presented. Finally, in section IV] we provide some further discussion of our results and allude to potential issues of our methods. Furthermore, we speculate about the implications of our findings to the rotating, asymptotically flat case.

\section{STATIONARY SOLITONS OF THE EMCKG SYSTEM IN A CAVITY}

\section{A. The framework}

We consider the following action, describing the EMcKG system (here we follow precisely the same conventions as in 20, 21, which allows a direct comparison with the results therein): $S=\int \sqrt{-g} \mathcal{L} d^{4} x$ with

$$
\mathcal{L}=\frac{R}{2}-\frac{1}{4} F_{\mu \nu} F^{\mu \nu}-\frac{1}{2} g^{\mu \nu} D_{(\mu}^{*} \Phi^{*} D_{\nu)} \Phi-\frac{\mu^{2}}{2}|\Phi|^{2},
$$

where we have used standard notation: $g$ is the metric determinant, $R$ is the Ricci scalar, $\mu$ is the scalar field mass and round brackets denote symmetrization, $Y_{(\mu \nu)}=\frac{1}{2}\left(Y_{\mu \nu}+Y_{\nu \mu}\right)$ for a tensor field $Y_{\mu \nu}$. We use a mostly positive space-time signature, and units in which $8 \pi G=c=1$. The scalar field $\Phi$ is complex, and $\Phi^{*}$ is the complex conjugate of $\Phi$. The electromagnetic field strength is denoted $F_{\mu \nu}=\nabla_{\mu} A_{\nu}-\nabla_{\nu} A_{\mu}$, where $A_{\mu}$ is the electromagnetic potential. In (1), we have introduced the gauge covariant derivative, $D_{\mu}=\nabla_{\mu}-i q A_{\mu}$, where $\nabla_{\mu}$ is the covariant derivative and $q$ is the scalar field charge. These conventions are slightly different from the ones in our previous works [1, 2]. We shall further remark on this in section III. Observe that the EMcKG system is invariant under the gauge transformation:

$$
A_{\mu} \rightarrow \tilde{A}_{\mu}=A_{\mu}+\partial_{\mu} \chi(x), \quad \Phi \rightarrow \tilde{\Phi}=e^{i q \chi(x)} \Phi,
$$

for a real function $\chi(x)$.

\section{B. The solitons}

To obtain the stationary solitonic solutions [20, 21], we shall consider an ansatz describing spherical, timeindependent configurations. For an isotropic coordinate system, the metric ansatz reads

$$
d s^{2}=-f_{0}(r) d t^{2}+f_{1}(r)\left[d r^{2}+r^{2}\left(d \theta^{2}+\sin ^{2} \theta d \varphi^{2}\right)\right],
$$

while the matter fields are

$$
A=A_{0}(r) d t, \quad \Phi=\phi(r) .
$$

In the following we shall be working with ansatz (3)-(4). But we observe that the gauge transformation (2), with $\chi=-\omega t / q$ yields the fields:

$$
\tilde{A}=\left(A_{0}(r)-\frac{\omega}{q}\right) d t, \quad \tilde{\Phi}=e^{-i \omega t} \phi(r) .
$$

In this gauge the scalar field oscillates with frequency $\omega$. We recall that the critical frequency for superradiance, $\omega_{c}$, around a $\mathrm{RN} \mathrm{BH}$, is set by the horizon electric potential as $\omega_{c}=q \phi\left(r_{H}\right) \equiv-q \tilde{A}_{0}\left(r_{H}\right)$, where $r_{H}$ is the radial coordinate of the horizon. This is the condition obeyed by a $\mathrm{BH}$ at the threshold of superradiance, which in terms of the 'old' gauge (4) reads $A_{0}\left(r_{H}\right)=0$.

With our metric ansatz the EMcKG equations reduce to a set of four second order equations plus a constraint (which is one of the Einstein eqs.). For solitons, these equations are solved starting with the following small- $r$ expansion

$$
\begin{aligned}
& f_{0}(r)=f_{00}+\frac{1}{6}\left(2 a_{0}^{2} q^{2}-f_{00} \mu^{2}\right) f_{10} \phi_{0}^{2} r^{2}+\mathcal{O}\left(r^{4}\right) \\
& f_{1}(r)=f_{10}-\frac{f_{10}^{2}}{12 f_{00}}\left(a_{0}^{2} q^{2}+f_{00} \mu^{2}\right) \phi_{0}^{2} r^{2}+\mathcal{O}\left(r^{4}\right) \\
& A_{0}(r)=a_{0}+\frac{1}{6} a_{0} f_{10} q^{2} \phi_{0}^{2} r^{2}+\mathcal{O}\left(r^{4}\right) \\
& \phi(r)=\phi_{0}-\frac{f_{10}}{6 f_{00}}\left(a_{0}^{2} q^{2}-f_{00} \mu^{2}\right) \phi_{0} r^{2}+\mathcal{O}\left(r^{4}\right)
\end{aligned}
$$

with $f_{00}, f_{10}, a_{0}, \phi_{0}$ four arbitrary constants and $f_{00}, f_{10}$ strictly positive such that the metric has the correct signature. In the numerical integration one takes $f_{00}=$ $f_{10}=1$. Then a continuum of solutions is found by varying the remaining parameters $a_{0}, \phi_{0}$ together with $q, \mu$.

Following [20] we choose $q=0.1$. We shall also focus on the massless scalar field case $\mu=0$, but some remarks about the massive case will be made in section IV The field equations are integrated numerically to find solitonic solutions by using a standard Runge-Kutta method. We start the numerical integration at $r=\epsilon$, where $\epsilon$ is typically $10^{-8}$, using the expansions (6) as initial conditions. One finds that the scalar field $\phi$ oscillates about zero (see Fig. 11; the mirror defining the cavity can be placed at any zero of the scalar field, but here we shall always consider the mirror to be located at the first zero of the scalar field, whose radial position is labelled $r=r_{m}$. Thus, in this model, for the stationary solutions, the mirror is "virtual" rather than corresponding to a concrete physical object that impacts on the EMcKG equations.

In section III we shall evolve eleven initial data sets, namely one which, according to [20, is associated with 

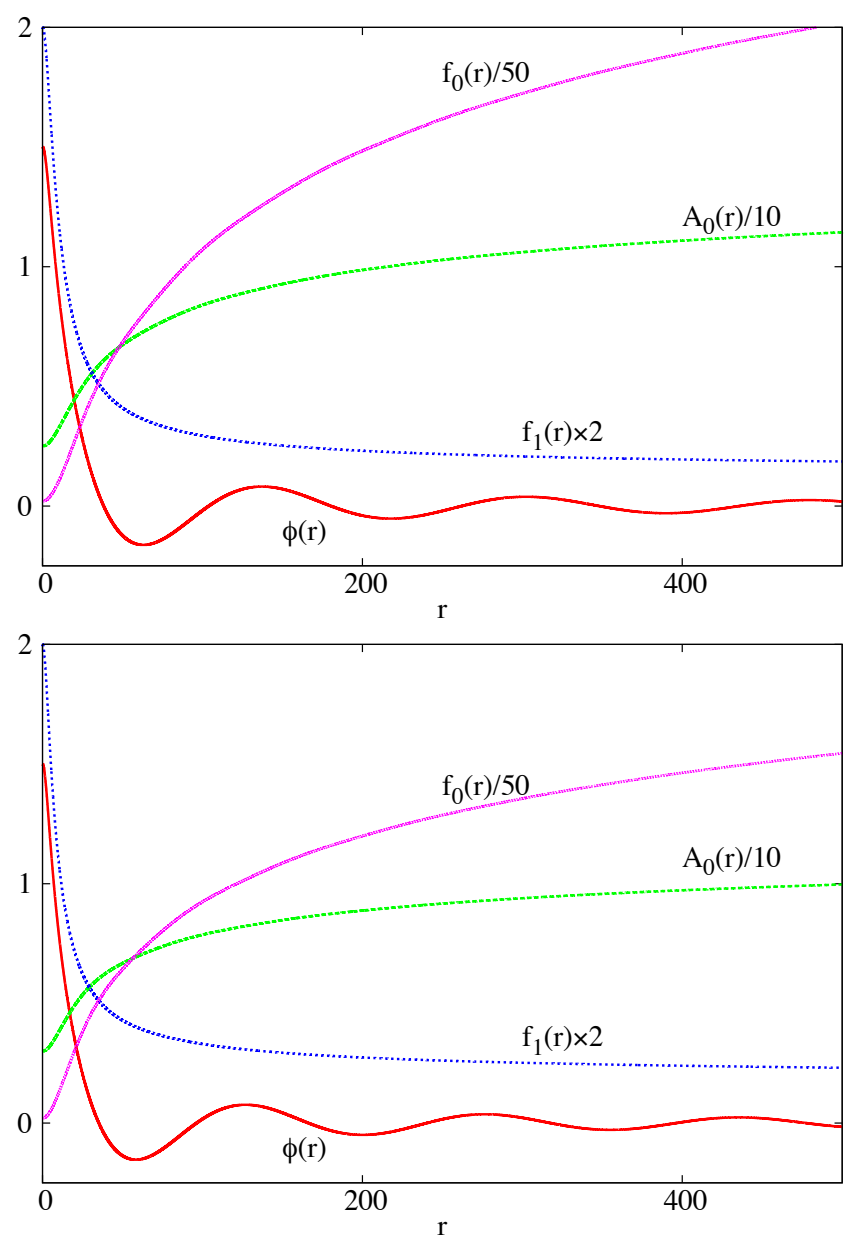

FIG. 1: Metric, scalar field and gauge field functions for model 1 (top panel) and model 5 (bottom panel). Some functions are rescaled for better visualization. Observe the oscillations of the scalar field. The mirror position is at the first zero of the scalar field (see Table I).

a stable solution (Model 0) and ten which are associated with unstable solitons (Models 1-10), all with $q=0.1$ and $\mu=0 .^{2}$ These models are summarized in Table I. where we also anticipate the outcome of their numerical evolution using our two schemes. To exemplify the behaviour of the various functions $\left\{f_{0}(r), f_{1}(r), \phi(r), A_{0}(r)\right\}$ for typical solitons, we display in Fig. 1 their radial dependence for models 1 and 5 in Table I. According to the results of [20] these are both unstable solitons. Indeed, as we discuss below, we see them evolving in both schemes. In particular, for scheme I, one of these will be shown to decay into a hairy $\mathrm{BH}$ (model 1) and the other to decay into a bald $\mathrm{BH}$ (model 5).

\footnotetext{
2 That these are stable or unstable can be observed by examining Fig. 11 in 20.
}

\section{NUMERICAL EVOLUTIONS}

\section{A. The framework}

The numerical evolutions of our initial data are performed in the EMcKG system (1) but, following our previous work [1, 2], we use slightly different conventions, corresponding to the following rescaling of the fields shown in (1):

$$
A_{\mu} \longrightarrow \sqrt{2} A_{\mu}, \quad \Phi \longrightarrow \sqrt{8 \pi} \Phi, \quad q \longrightarrow \frac{q}{\sqrt{2}} .
$$

The metric and mass parameter are unchanged. Rescalings (7) are imposed to all the data in Table I, which the table reports before the rescaling. This change also ensures that we use the same values for $a_{0}, \phi_{0}$ and $q$ than in [20].

The time update of the different systems of evolution equations we have to solve in our code (Einstein, KleinGordon, and Maxwell) is done using the same type of numerical techniques we have extensively used in previous work (see, in particular, 2, 28, 30). We refer the interested reader to those references for full details. Here, we simply mention that the evolution equations are integrated using the second-order PIRK method developed by [31, 32]. This method allows to handle the singular terms that appear in the evolution equations due to our choice of curvilinear coordinates. The derivatives in the spacetime evolution are computed using a fourth-order centered finite-difference approximation on a logarithmic radial grid. We also use fourth-order Kreiss-Oliger dissipation to avoid high-frequency noise appearing near the outer numerical boundary. For the simulations presented in this work we evolve the electric field explicitly and the electric potentials implicitly.

We perform the evolution of the unstable and the stable solitons by rescaling the lapse function of the $3+1$ formalism [33] in the following way:

$$
\tilde{\alpha}=\alpha / \alpha\left(r_{\max }\right) .
$$

where $\alpha=\sqrt{f_{0}}$. The lapse is rescaled to facilitate our numerical simulations, as it ensures that $\tilde{\alpha} \rightarrow 1$ at the outer boundary of our grid, which corresponds to Minkowski spacetime.

\section{B. The two schemes}

We are interested in the dynamics of the solitons inside the cavity bounded by the mirror at $r=r_{m}$. Whereas the presence of the mirror in the stationary soliton solution is a matter of perspective, its presence in a dynamical evolution must be enforced by some dynamical rule. In other words, since a generic evolution of the initial data provided by the solitons described in the previous section, will not preserve the node at $r=r_{m}$, one has to place 


\begin{tabular}{|c|c|c|c||c|c|c||c|c|c||c|c|c|}
\hline Model & $a_{0}$ & $\phi_{0}$ & $r_{m}$ & Scheme I & $\omega_{c}$ & $\omega_{\Phi}$ & Scheme II a & $\omega_{c}$ & $\omega_{\Phi}$ & Scheme II b & $\omega_{c}$ & $\omega_{\Phi}$ \\
\hline \hline 0 & 1.5 & 1.5 & 45.2 & HBH & 0.044 & 0.050 & Stable & & & Stable & & \\
\hline 1 & 2.5 & 1.5 & 38.2 & HBH & 0.057 & 0.056 & (bald) RN & - & - & (bald) RN & - & - \\
2 & 2.6 & 1.5 & 37.6 & HBH & 0.057 & 0.055 & (bald) RN & - & - & (bald) RN & - & - \\
3 & 2.7 & 1.5 & 37.0 & HBH & 0.058 & 0.055 & (bald) RN & - & - & (bald) RN & - & - \\
4 & 2.8 & 1.5 & 36.4 & HBH & 0.056 & 0.054 & (bald) RN & - & - & (bald) RN & - & - \\
5 & 3.0 & 1.5 & 35.2 & (bald) RN & - & - & (bald) RN & - & - & (bald) RN & - & - \\
\hline 6 & 3.0 & 1.8 & 56.8 & HBH & 0.040 & 0.039 & HBH & 0.004 & 0.004 & HBH & 0.004 & 0.004 \\
7 & 3.2 & 1.8 & 55.8 & HBH & 0.042 & 0.042 & HBH & 0.005 & 0.004 & HBH & 0.004 & 0.004 \\
8 & 3.4 & 1.8 & 54.8 & HBH & 0.043 & 0.042 & HBH & 0.006 & 0.005 & HBH & 0.004 & 0.004 \\
9 & 4.0 & 1.8 & 52.0 & HBH & 0.048 & 0.046 & (bald) RN & - & - & (bald) RN & - & - \\
10 & 4.5 & 1.8 & 49.8 & (bald) RN & - & - & (bald) RN & - & - & (bald) RN & - & - \\
\hline
\end{tabular}

TABLE I: The eleven soliton models we use as initial data, all with $q=0.1$ and $\mu=0$. From left to right the columns report: the model number, the parameters $a_{0}, \phi_{0}$ and $r_{m}$ characterizing the corresponding solitons (columns 2 to 4 ), and the outcome of the evolutions for the two schemes (following columns), in particular if a hairy $\mathrm{BH}(\mathrm{HBH}$ ) forms or if a bald RN BH results from the evolution. In the former case, the critical frequency of the $\mathrm{BH}, \omega_{c}$, and the corresponding frecuency of oscillation of the scalar field, $\omega_{\Phi}$, are also indicated.

a mirror at the location of the node, enclosing a cavity. Here we shall consider two different dynamical rules.

In Scheme I we shall use the initial data provided by the stationary soliton solutions only inside the cavity. That is, scheme I is based on a truncated soliton. Outside the cavity we change the initial data by setting the scalar field to zero all along the computational domain, but leaving the metric fields unchanged. Whereas this is the simplest thing to do, within the "scalar field enclosed in a cavity" perspective, it has the obvious shortcoming that the initial data outside the cavity does not satisfy the field equations. In other words, the initial data is constraint-violating outside the cavity. As we show below, however, the simulation converges to a solution of the field equations within acceptable errors for this system, the endpoint being either a hairy $\mathrm{BH}$ or a bald $\mathrm{BH}$, depending on the starting soliton. We remark that this dynamical perspective departs from that encoded in the perturbation theory considered in [20, where the soliton extends to spatial infinity and the perturbations are required to preserve the node at the mirror location. As a consequence of this difference, model 0 in Table 1 which according to 20] is a stable soliton, turns out to evolve in scheme I.

In Scheme II, on the other hand, we shall use the initial data provided by the stationary soliton solutions both inside and outside the cavity. We therefore use the full soliton. The presence of the mirror is enforced by not evolving the scalar field value at the mirror, which is initially put to zero. This ensures the presence of the node during the whole evolution and (within numerical error) the absence of scalar energy or charge flux between the inside and outside of the cavity. This enforcement leads to more localized constraint violations, which occur only at the mirror location, and to a closer correspondence with the perturbation problem discussed in 20. The results of the evolutions, however, are qualitatively similar to those of scheme I, i.e. we see two possible outcomes, but the "same" soliton in both schemes may have a different fate. In scheme II we perturb the soliton inside the cavity by multiplying $\Phi$ by a factor slightly larger than one in order to trigger the evolution; then we consider two different perturbation factors leading to two sub-schemes: scheme IIa, with a factor of 1.05 , and scheme IIb, with a factor of 1.01 .

Our simulations employ a logarithmic radial grid with a maximum resolution of $\Delta r=0.025 M$. The outer boundary of the computational domain is placed at $r_{\max }=4.5 \times 10^{3}$, far enough as to not affect the dynamics in the inner region during the entire extent of the simulations. The (Courant) time step is given by $\Delta t=0.1 \Delta r$ which ensures long-term stable simulations.

\section{Numerical results - scheme I}

Let us start by describing the numerical results under scheme I. In Fig. 2 (top panel) we show the result for the evolution of the energy in the scalar field computed as in 1, 2, for models 1-5. We observe that after an initial period of no remarkable change, all models exhibit a sharp decrease of the energy in the scalar field at $t \gtrsim$ 150. For models 1-4 what follows is a new, non-zero, equilibrium value of the scalar field energy. However, for model 5 the energy in the scalar field keeps decreasing and tends to vanish asymptotically.

In all models 1-5 we observe the formation of an apparent horizon $(\mathrm{AH})$ at a time which roughly coincides with the sharp decay of the scalar field energy visible in Fig. 2 . This is illustrated in the bottom panel of the figure for model 3. One can then compute the charge inside the $\mathrm{AH}$ and the charge in the scalar field using the expressions described in 1, 2. The result is exhibited in Fig. 3 for models 3 and 5 . A clear charge transfer from the scalar 

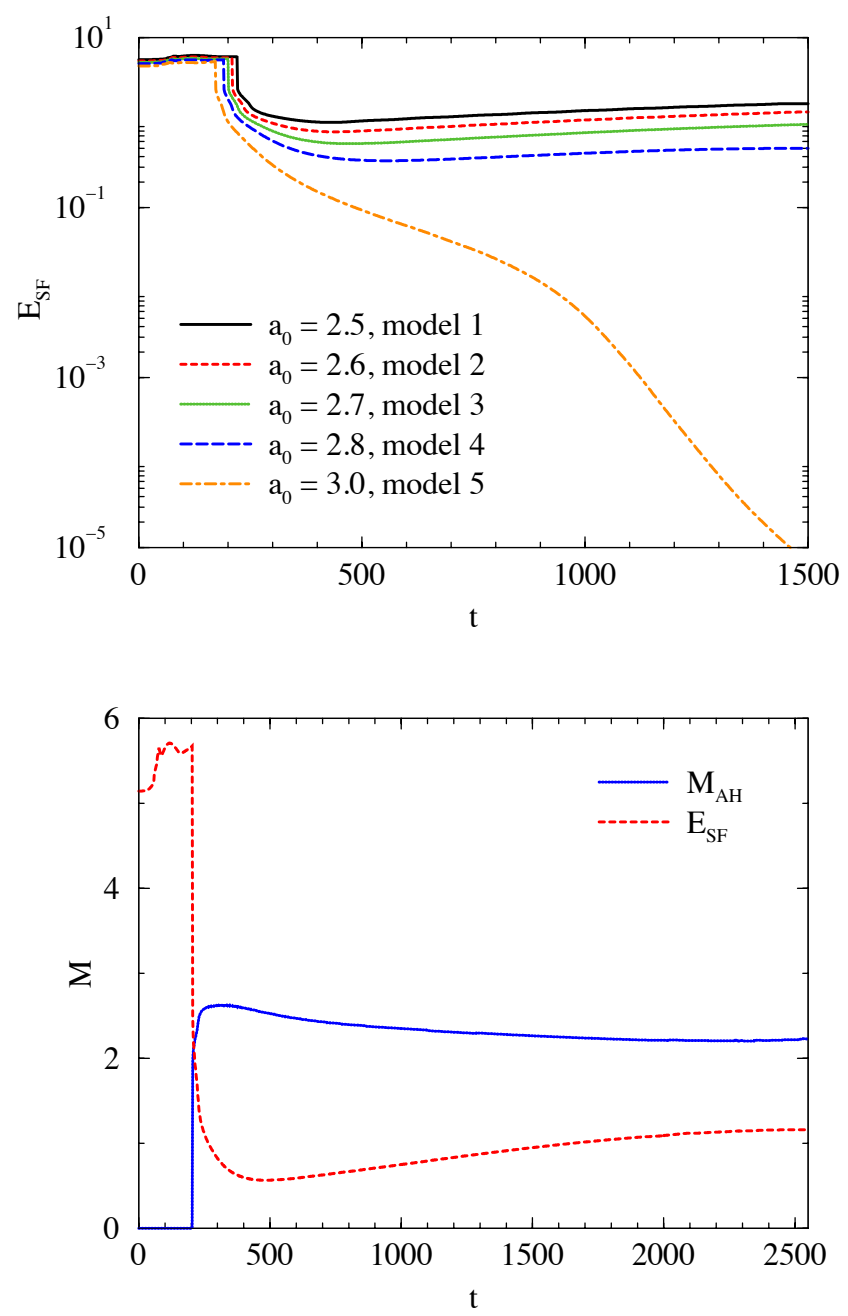

FIG. 2: Time evolutions in scheme I: the top panel shows the scalar field energy for models 1-5, while the bottom panel shows the AH mass and scalar field energy for model 3.

field (soliton) to the BH is observed, with the total charge (black solid line) remaining constant within an excellent approximation. Whereas in model 3 the charge in the scalar field remains non-zero signaling the formation of a hairy $\mathrm{BH}$, in model 5 the scalar field charge vanishes, signaling the formation of a (bald) $\mathrm{RN} \mathrm{BH}$.

To examine in more detail the type of hairy $\mathrm{BH}$ that forms in the decay of the soliton we show in the top panel of Fig. 4 the real part of the scalar field for models 1-5, as a function of time, extracted at a specific radial position $r=5$. Correspondingly, the bottom panel of Fig. 4 displays the real and imaginary parts of the scalar field for model 3 as an illustrative example. It can be seen that for all these models the real part is a sinusoidal function and that the real and imaginary parts have a phase difference of $\pi / 2$; therefore, they oscillate with an opposite phase. The critical frequency for the BH that forms and the frequency of oscillation of the scalar field, computed
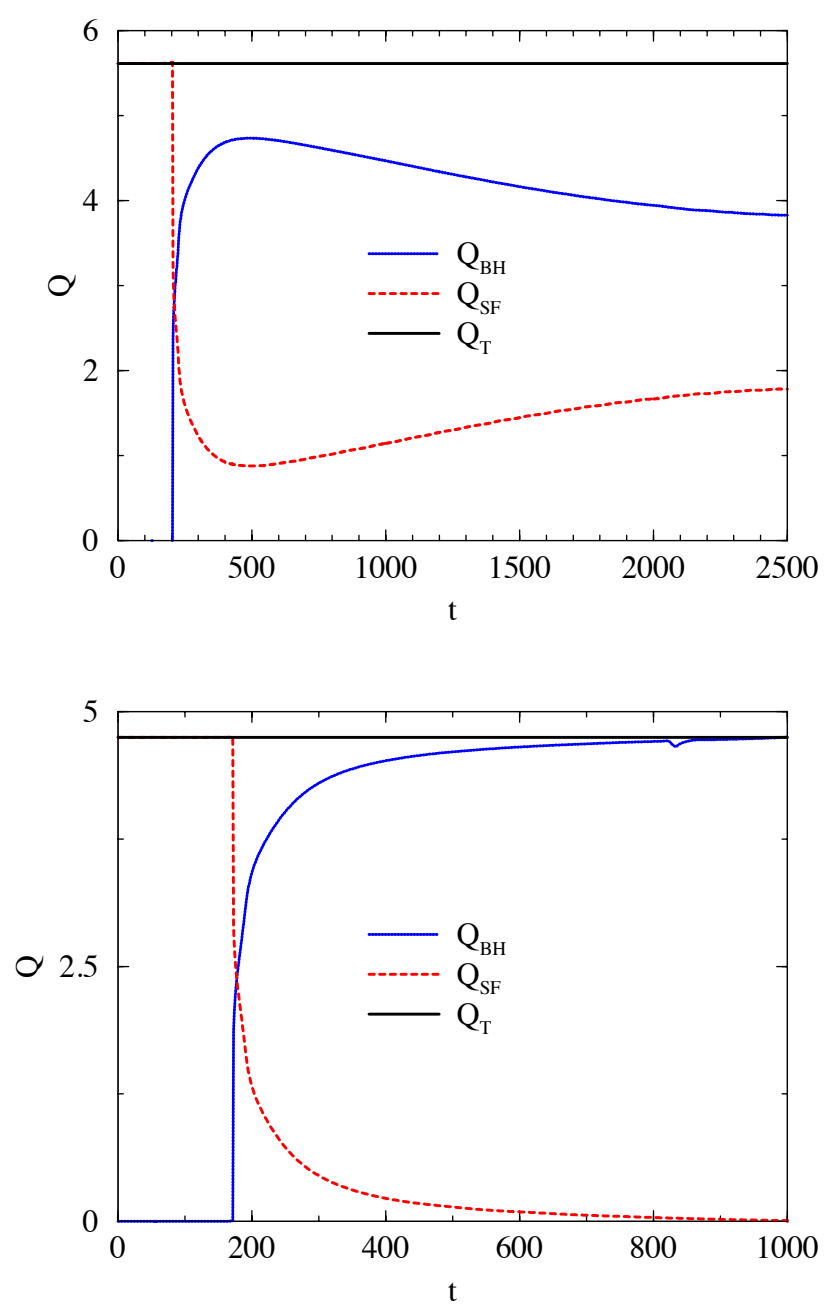

FIG. 3: Time evolution of the charge in the scalar field, $Q_{\mathrm{SF}}$, in the $\mathrm{BH}$ (computed at the $\mathrm{AH}), Q_{\mathrm{BH}}$, and of the total charge, $Q_{\mathrm{T}}$, for models 3 (top panel) and 5 (bottom panel), in scheme I.

as in 1, 2] are shown in Table I. They match within the numerical error, showing that these BHs are hairy BHs at the threshold of superradiance.

Finally, we display in Fig. 5 the radial profile of the scalar field magnitude for model 3 and at different times of the evolution. It is worth noticing that the maximum value attained by the scalar field (best visible in the inset) is inside the $\mathrm{BH}$ horizon (signaled by the dashed vertical line). Therefore, the scalar field decays monotonically from the horizon to the mirror, as one expects for stable hairy BHs [1, 9].

The results we have just discussed establish that a hairy $\mathrm{BH}$ can form as the outcome of numerical evolutions of unstable solitons. There are, however, two issues that must be discussed in the current scheme. The first issue is related to the constraint violations of the simulations. These arise due to the choice of initial data in 

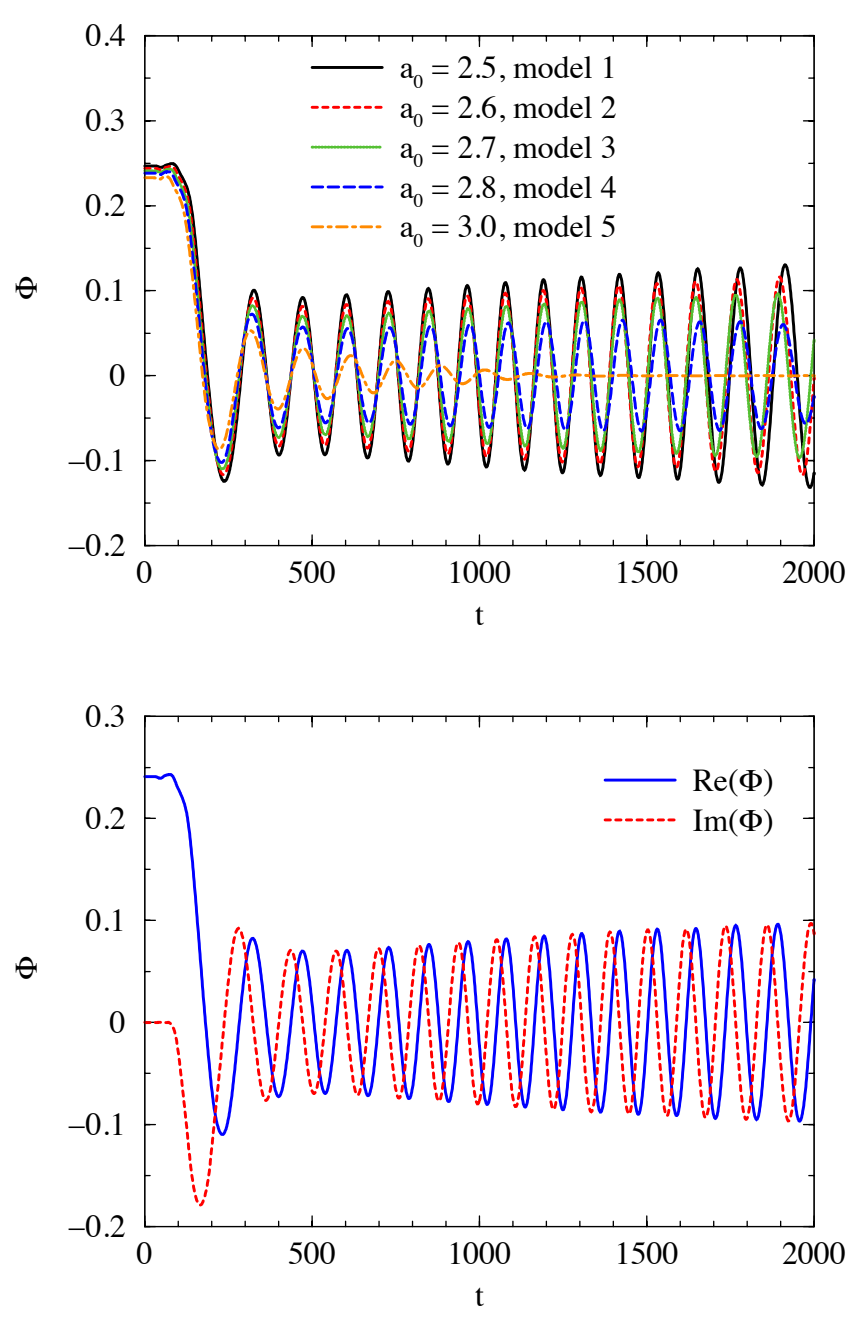

FIG. 4: Time evolution of the real part of the scalar field amplitude for models 1-5 (top panel) and of the real (blue solid line) and imaginary (red dashed line) parts of the scalar field amplitude for model 3 (bottom panel). Data are extracted at $r=5$. Notice the decay of the field amplitude in model 5 .

scheme I, in which we set the scalar field to zero outside the mirror, but also due to the coordinate singularity at $r=0$. Figure 6 plots the time evolution of the L2 norm of the Hamiltonian constraint for model 3 for three different radial grid resolutions. Whereas relatively large constraint violations are observed at some early phase of the evolution, namely during the formation of the $\mathrm{AH}$ and shortly after, the values of the violations decrease significantly for the remaining part of the evolution. We note that the values attained in the final state are comparable to those observed in our previous works [1, 2].

The second issue is related to the stable solution model 0 in Table I We have checked that the time evolution of this model indicates it is not stable; rather it evolves. What concerns us here is not so much what is the endpoint of this evolution, but the fact that the soli-

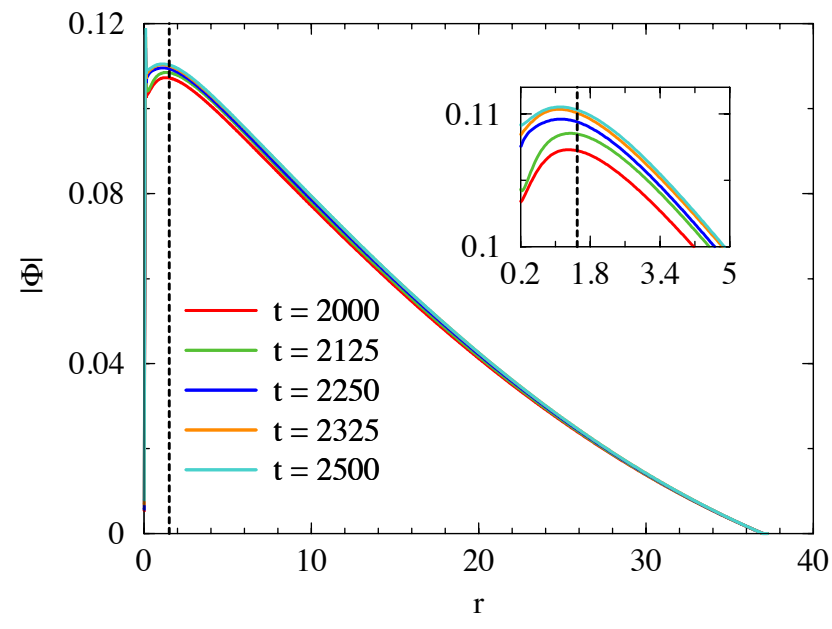

FIG. 5: Radial profile of the scalar field magnitude for model 3 at different times of the evolution with scheme I. The vertical dashed line marks the location of the $\mathrm{AH}$.

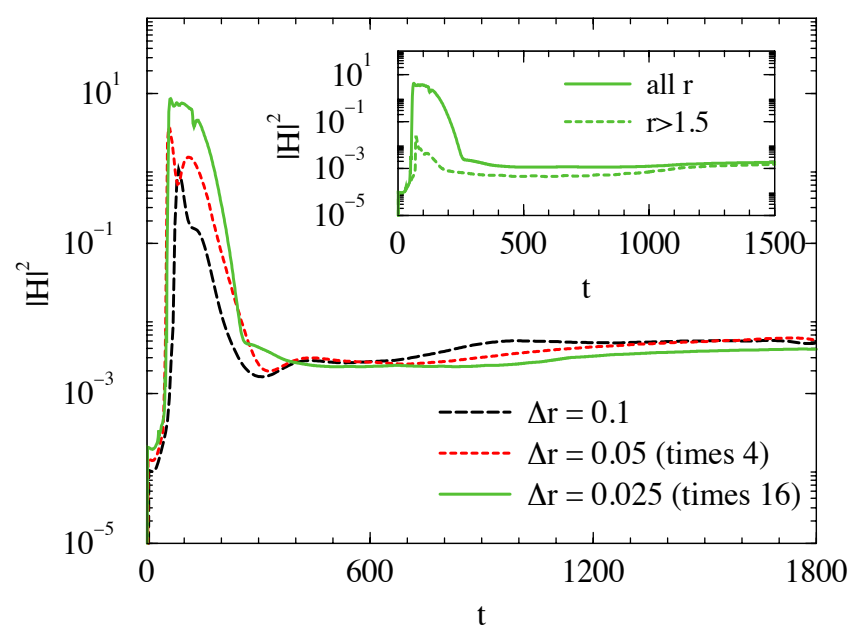

FIG. 6: Time evolution of the L2 norm of the Hamiltonian constraint for model 3 using scheme I. The inset shows the evolution when considering the entire radial domain (solid line) and when removing the radial zones that eventually lie within the AH (dashed line). Second-order convergence is approximately achieved in the evolution for $t \gtrsim 400$.

ton in our dynamical scheme I is not stable, whereas it is stable in the perturbation scheme considered in [20, 21]. This discrepancy underlines the fact that the dynamical perturbations considered therein are not faithfully represented by our scheme I, and leads us to consider scheme II below.

To argue the generality of the behaviour observed for models 1-5 under scheme I, we display in figure 7 the time evolution of the scalar field energy (top panel) and the oscillations of the real part of the scalar field (bottom 

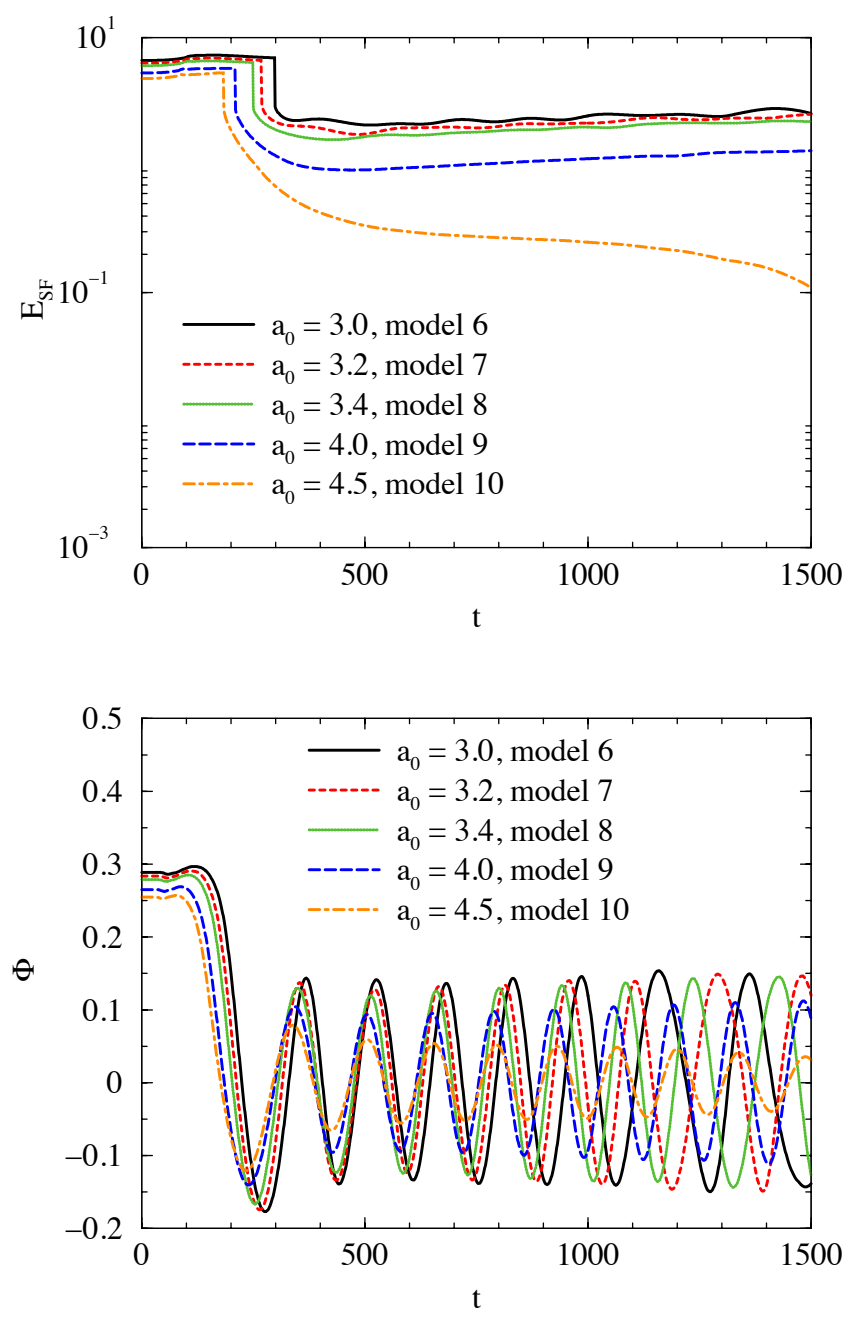

FIG. 7: Time evolution of the scalar field energy (top panel) and of the real part of the scalar field amplitude (bottom panel) for models $6-10$ in scheme I. The extraction radius is at $r=5$.

panel) for models 6-10. We observe the same trend to that found in models 1-5, namely, for the same value of $\phi_{0}$ sufficiently small values of $a_{0}$ lead to a hairy $\mathrm{BH}$, but for larger values the final state changes and a bald $\mathrm{RN}$ $\mathrm{BH}$ is obtained instead. Model 10 is the only one whose energy decreases during the rest of the evolution after the $\mathrm{BH}$ is formed. The total amount of scalar field energy remaining in the cavity is larger than for the previous five models.

\section{Numerical results - scheme II}

We now turn our attention to scheme II. In Fig. 8 we plot the evolution of the scalar field energy, for models 010 within scheme IIa (the first six models in the top panel and the last five in the bottom one). The first observation
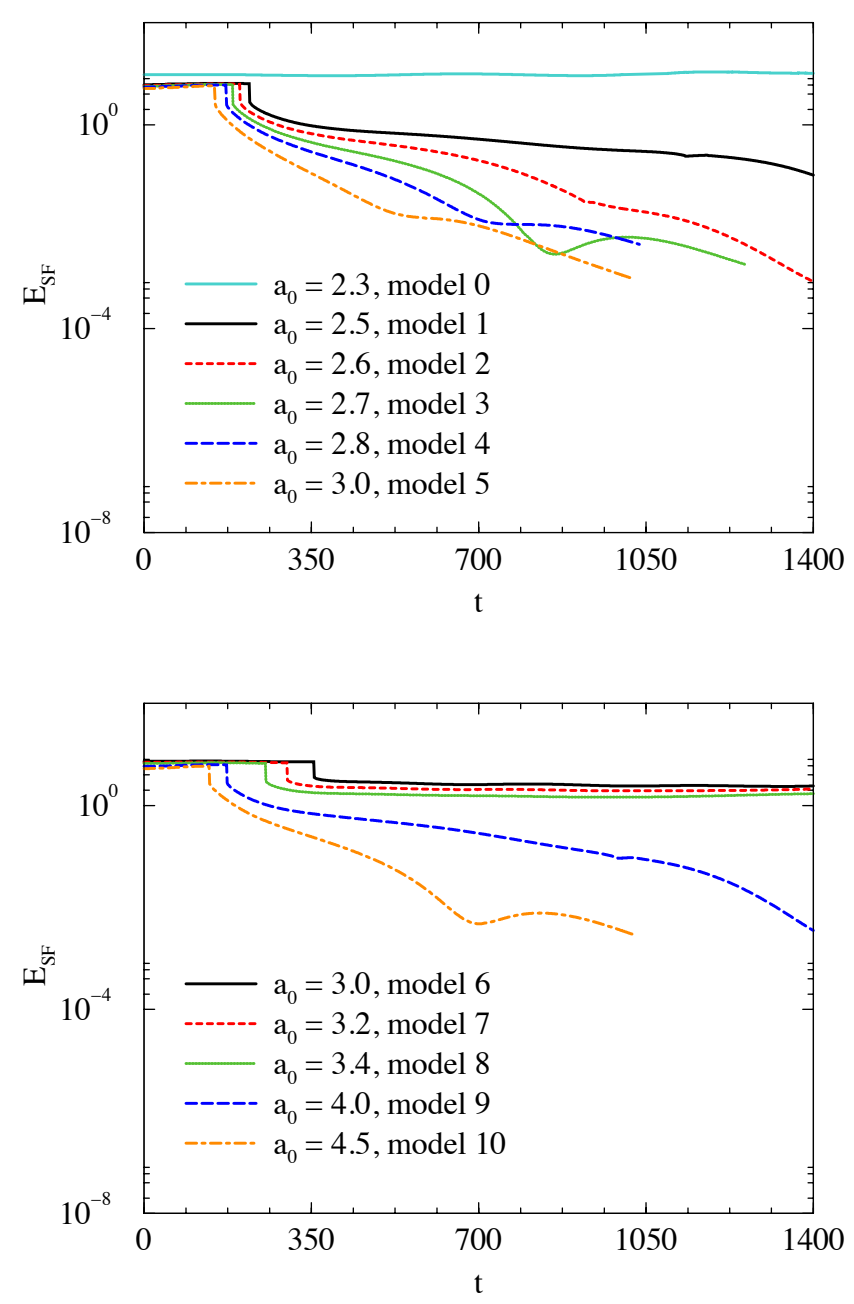

FIG. 8: Time evolutions of the scalar field energy for models 0-5 (top panel) and models 6-10 (bottom panel), using scheme IIa.

is that model 0 is now stable, in accordance to [20], and the energy remains almost constant, taking into account that the soliton is slightly perturbed. For the unstable models 1-5, a $\mathrm{BH}$ forms at $t \gtrsim 200$ absorbing part of the scalar field energy. For all these models, the energy in the scalar field keeps decreasing with time, tending to vanish rather than approaching an equilibrium state. A different result is found for models 6-8, displayed in the bottom panel of Fig. 8. For these models the scalar field energy is almost constant after the formation of the BH. As observed in scheme I, however, increasing $a_{0}$ for fixed $\phi_{0}$ beyond a certain value, the scalar field energy keeps decreasing with time, indicating that a bald RN $\mathrm{BH}$, rather than a hairy $\mathrm{BH}$, forms (cf. models 9 and 10 in the bottom panel of Fig. 8).

The charge transfer from the scalar field (soliton) to the $\mathrm{BH}$ in scheme II is monitored in Fig. 9, where we plot the evolution of the charge of the $\mathrm{BH}$, of the scalar 


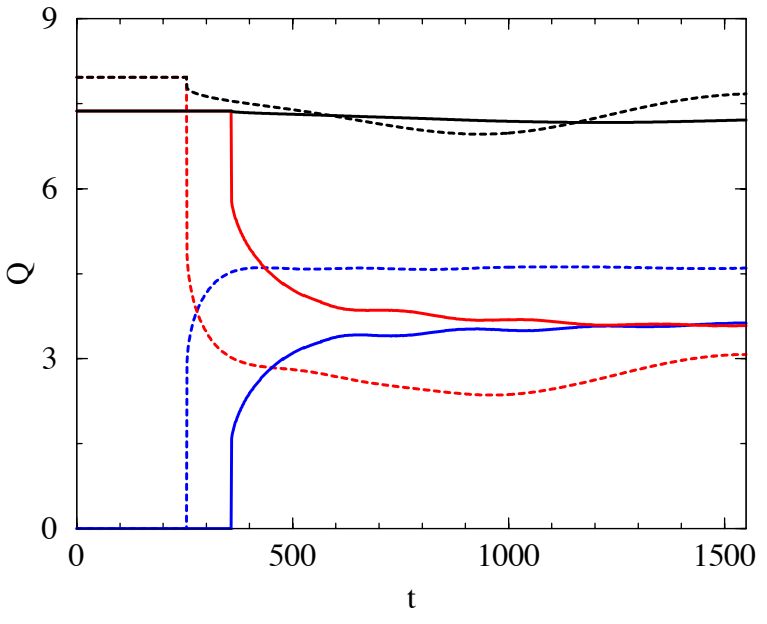

FIG. 9: Time evolution of the charge in the scalar field, $Q_{\mathrm{SF}}$ (red curves), in the $\mathrm{BH}$ (computed at the $\mathrm{AH}), Q_{\mathrm{BH}}$ (blue curves), and of the total charge $\left(Q_{\mathrm{T}}\right)$ (black curves), for model 8. Dashed curves correspond to scheme IIa (5\% perturbation) and solid lines to scheme IIb (1\% perturbation).

field, and of the total charge, for model 8 and for the two perturbations considered (schemes IIa and IIb). A clear charge transfer can be seen from the scalar field to the $\mathrm{BH}$ at the time of formation of the $\mathrm{AH}$. The total charge, however, decreases when the $\mathrm{BH}$ forms (black curves), signaling an issue. This is in fact due to the amount of perturbation we set up initially. Comparison of both schemes shows that when the initial perturbation to the solution is reduced from $5 \%$ (scheme IIa, dashed black lines) to $1 \%$ (scheme IIb, solid black lines), then total charge conservation holds at a higher accuracy. Using a large perturbation has the desirable feature that it triggers faster dynamics and hence leads to shorter simulations; however, as this example shows, too large perturbations introduce also undesirable features in the dynamics of the system, as total charge variation.

In Fig. 10, we show the scalar field amplitude extracted at $r=5$ for model 8 for scheme IIb. As before we find that the real and imaginary parts of the scalar field oscillate with opposite phases. Moreover, as shown in Table I. the oscillation frequency of the scalar field matches, to a good accuracy, the critical frequency, establishing the formation of a hairy $\mathrm{BH}$ at the threshold of superradiance. We observe that, for scheme IIb, the oscillation frequency is an order of magnitude smaller than in scheme I, so we can only capture roughly one period. We note that this already required using a larger computational grid than for the simulations based on scheme I.

Let us focus now in the stable solution, model 0 . We have already shown in Fig. 8 that the evolution of the scalar field energy in this model is stable in scheme II, contrary to what we found when using scheme I. To add further proof of its stable character we plot in Fig. 11

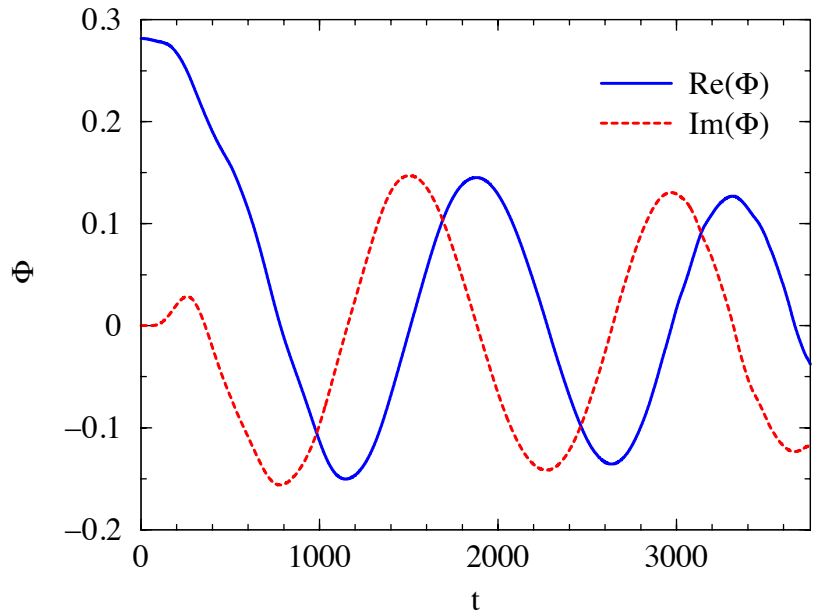

FIG. 10: Time evolution of the real (blue solid line) and imaginary (red dashed line) parts of the scalar field amplitude for model 8 and scheme IIb. Data are extracted at $r=5$.

the radial profile of the scalar field magnitude for different times for model 0 without initial perturbation (top panel) and the evolution of the scalar field charge (bottom panel). These results show that this model is indeed stable during our numerical evolutions with scheme II. The solution remains essentially invariant, up to numerical error and the constraint violations induced by the presence of the mirror.

Finally, in Fig. 12 we show the evolution of the L2 norm of the Hamiltonian constraint for scheme IIa for model 8, for three radial grid spacings. We observe that although there is an initial growth of the Hamiltonian constraint violation, similarly to what happens with scheme I and also around the time the black hole forms, this violation now reaches a lower maximum than that observed in scheme I. Moreover, as in scheme I, the violations are significantly dissipated for $t \gtrsim 400$ and the evolution drives the values of the constraints to acceptable levels. Finally, the analysis with various grid spacings shows that the convergence order of the code is second order to reasonable accuracy, as expected.

\section{DISCUSSION}

The endpoint of the superradiant instability of the Kerr BH, triggered by a confined bosonic scalar field, remains as an important open question in the theoretical understanding of $\mathrm{BHs}$ - see 34] for a review of superradiance. In particular, the discovery of new stationary BH solutions - Kerr BHs with scalar [11, 12] or Proca hair [13] - that exist at the threshold of superradiance, raises the question if these could be endpoints of the instability.

As a simpler model for this problem, with important 

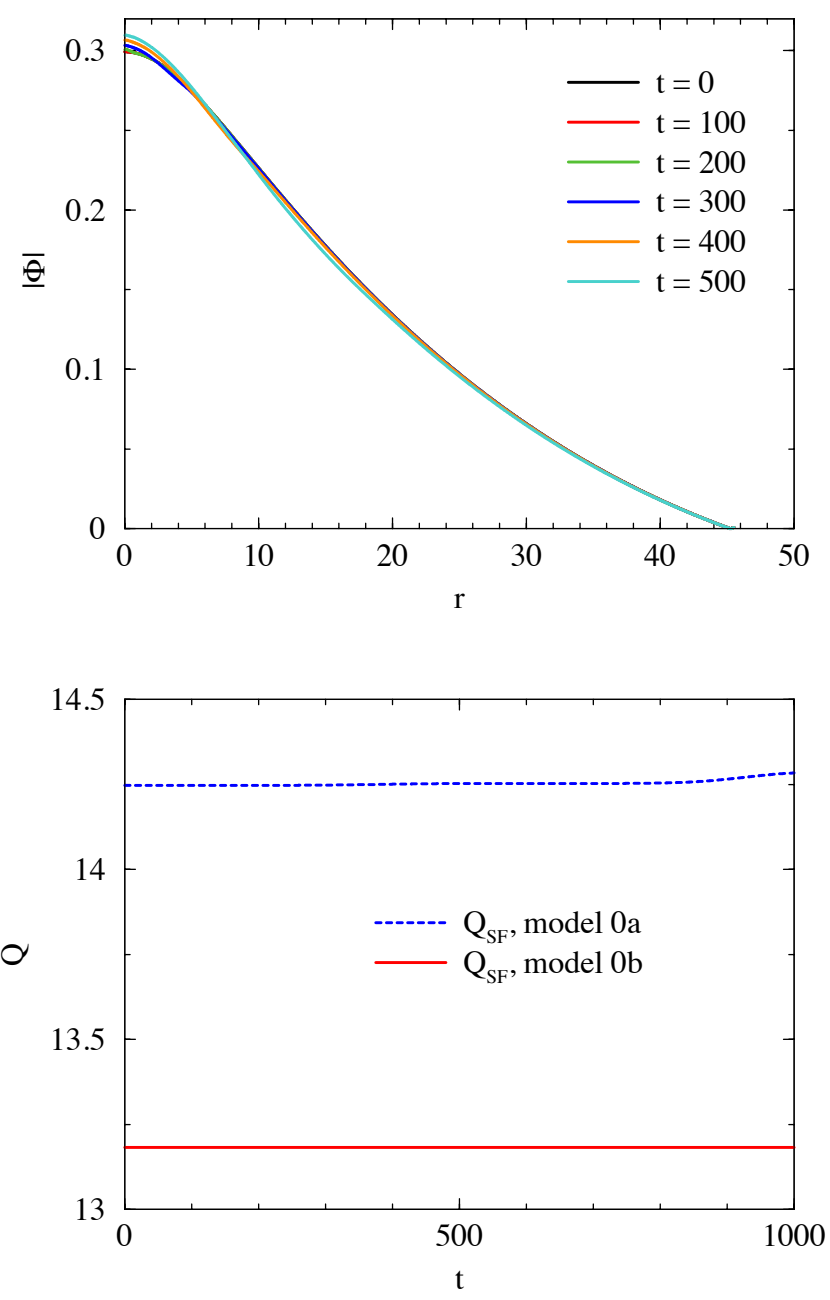

FIG. 11: (Top panel): Radial profile of the scalar field magnitude for model 0 without adding a perturbation and for different times. (Bottom panel) : Time evolution of the scalar field charge for models 0a (blue dashed line) and 0b (red solid line). The total charge variation observed for model 0a towards the end of the simulation is related to the use of much too large a perturbations, as discussed in the main text.

technical advantages, recent studies of the fully nonlinear evolution of the superradiant instability have considered the EMcKG system and focused on the case of a RN BH enclosed in a cavity 1, 2, which is also superradiantly unstable in the presence of a charged scalar field $4-8 .{ }^{3}$ The dynamical evolution of this unstable system has been shown to lead to the formation of hairy BHs at the threshold of superradiance. These BHs were found in 9 (see also [10), and can be faced as the analogues, in this system, of Kerr BHs with scalar hair [11, 12.

\footnotetext{
${ }^{3}$ See [35] for the non-linear evolution of a superradiantly unstable RN BH in asymptotically Anti-de-Sitter spacetime.
}

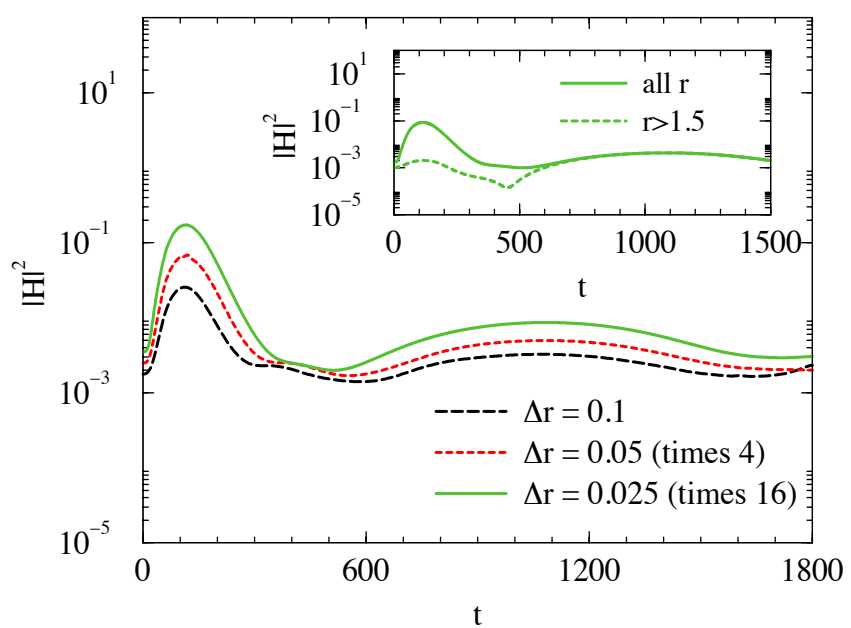

FIG. 12: Time evolution of the L2 norm of the Hamiltonian constraint for model 8 using scheme IIa. The inset shows the evolution for the entire radial domain (solid line) and for $r>1.5$ (dahsed line), thus removing the radial zones that eventually lie within the $\mathrm{AH}$.

The existence of charged scalar solitons in this same system, recently reported in 20, 21, some of which are unstable, raises the question about the endpoint of these unstable solitons. Understanding this question has been the main motivation of this paper. We have tackled the question by considering two different dynamical schemes, that impose the confinement rule for the scalar field in the cavity differently. Our main conclusion is that, albeit there are quantitative differences between these two schemes - most notably, the end state of some of our models is a hairy $\mathrm{BH}$ when using scheme I, and a bald $\mathrm{RN} \mathrm{BH}$ when using scheme II -, the main message is similar. Indeed, we observe the same pattern for both schemes: there seems to be a critical value of $a_{0}$ for a given $\phi_{0}$ below which the collapse of unstable solitons leads to a hairy $\mathrm{BH}$ rather than to a bald RN BH. Models 6-10 in particular provide a good example. The value of the critical $a_{0}$ differs, but we see the two possibles outcomes for both schemes; for scheme I the transition occurs from model 9 to 10, whereas for scheme II it occurs from model 8 to 9 . In a nutshell, we believe our numerical results provide evidence that there is a second channel for the dynamical formation of a hairy $\mathrm{BH}$ at the threshold of superradiance: from the decay of certain unstable solitons. The first channel is, of course, the aforementioned growth of the superradiant instability.

There are a number of interesting physical questions in this system, which our study raises, for instance:

1) is there a simple criterion/physical understanding separating the decay into a hairy $\mathrm{BH}$ vs a bald $\mathrm{BH}$ ? We have observed that for a fixed value of $\phi_{0}$ sufficiently small (large) values of $a_{0}$ produce a hairy 
(bald) BH. Can one get a deeper understanding?

2) There are some stable hairy BHs that cannot form through the first channel, as they do not obey the $\mu<q$ superradiance requirement [5] or even a stronger lower bound [8] - see the discussion in [21]. Can these form from the evolution of an unstable soliton? More generically, what is the impact of the scalar field mass $\mu$ on our results?

3) The hairy BHs that form through the "old" channel (superradiant growth around a bald $\mathrm{BH}$ ) are not very hairy, in the sense that the mass of the scalar configuration is not comparable to the mass of the $\mathrm{BH}$. Could very hairy BHs, with almost all of the energy in the hair, form from the decay of unstable solitons?

Whereas all these questions are certainly worthwhile to address, our study has also exposed some frailties of this system that raise doubts on how much more one can pursue it, without introducing further complexity. The scalar-field-confining mirror did not impose important challenges when addressing test scalar field evolutions $[4,8]^{4}$, or fully non-linear stationary solutions [9, 10, 20, 21]. When making fully non-linear dynamical evolutions, however, the "virtual" mirror becames a source of constraint violations. In our previous simulations 1, 2, the scalar field was initialized with very small values and never became too large. In the case under study here, however, the scalar field is large from the outset, as it describes a self-gravitating scalar soliton, which makes the mirror a more important source of numerical error and, consequently, makes the evolutions more challenging. Moreover, in the horizonless case, the coordinate singularity (of spherical coordinates) at the origin also sources constraint violations. Fortunately, we have observed that the system evolves towards acceptable values of the constraint violations.

We close by returning to the parallelism with the asymptotically flat case. The "cousin" solutions to the solitons studied herein are the well known boson stars 22, self-gravitating systems formed by massive scalar fields (eventually, but not necessarily, selfinteracting). There are stable and unstable boson stars. Stable ones undergo oscillations when perturbed and show similar properties to other compact objects, such as neutron stars. Unstable boson stars, however, may collapse into BHs, radiate the energy excess or completely disperse away. These behaviours have been explicitly shown by numerical evolutions for spherically symmetric boson stars [44 48. In that case, however, there are

\footnotetext{
${ }^{4}$ For test fields, in fact, the confining mirror has been widely used in other studies of fields interacting with charged BHs, see e.g. 36, 43.
}

no hairy BHs [49, which require rotation and thus exist only in axisymmetry [27. Thus, in a similar spirit to the results in this work, could unstable rotating boson (or Proca) stars form Kerr BHs with scalar (or Proca) hair? This is an interesting open question.

\section{Acknowledgements}

This work has been supported by the Spanish MINECO (AYA2013-40979-P), by the Generalitat Valenciana (PROMETEOII-2014-069), by the CONACyT-SNI-México, by the FCT (Portugal) IF programme, by the CIDMA (FCT) strategic project UID/MAT/04106/2013 and by the EU grants NRHEP295189-FP7-PEOPLE-2011-IRSES and H2020-MSCARISE-2015 Grant No. StronGrHEP-690904. Computations have been performed at the Servei d'Informàtica de la Universitat de València and at the Blafis cluster at the University of Aveiro. 
[1] N. Sanchis-Gual, J. C. Degollado, P. J. Montero, J. A. Font, and C. Herdeiro, Phys. Rev. Lett. 116, 141101 (2016), 1512.05358.

[2] N. Sanchis-Gual, J. C. Degollado, C. Herdeiro, J. A. Font, and P. J. Montero, Phys. Rev. D94, 044061 (2016), 1607.06304.

[3] S. Hod, Phys. Lett. B713, 505 (2012), 1304.6474.

[4] S. Hod, Physical Review D 88, 064055 (2013), 1310.6101.

[5] C. A. R. Herdeiro, J. C. Degollado, and H. F. Rnarsson, Phys.Rev. D88, 063003 (2013), 1305.5513.

[6] J. C. Degollado and C. A. R. Herdeiro, Phys.Rev. D89, 063005 (2014), 1312.4579.

[7] S. Hod, Phys. Lett. B747, 339 (2015), 1507.01943.

[8] S. Hod, Phys. Lett. B755, 177 (2016), 1606.00444.

[9] S. R. Dolan, S. Ponglertsakul, and E. Winstanley, Phys. Rev. D92, 124047 (2015), 1507.02156.

[10] P. Basu, C. Krishnan, and P. N. B. Subramanian (2016), 1609.01208.

[11] C. A. R. Herdeiro and E. Radu, Phys.Rev.Lett. 112, 221101 (2014), 1403.2757.

[12] C. Herdeiro and E. Radu, Class. Quant. Grav. 32, 144001 (2015), 1501.04319.

[13] C. Herdeiro, E. Radu, and H. Runarsson, Class. Quant. Grav. 33, 154001 (2016), 1603.02687.

[14] B. Kleihaus, J. Kunz, and S. Yazadjiev, Phys. Lett. B744, 406 (2015), 1503.01672.

[15] C. A. R. Herdeiro, E. Radu, and H. Rnarsson, Phys. Rev. D92, 084059 (2015), 1509.02923.

[16] J. F. M. Delgado, C. A. R. Herdeiro, E. Radu, and H. Runarsson, Phys. Lett. B761, 234 (2016), 1608.00631.

[17] O. J. C. Dias, G. T. Horowitz, and J. E. Santos, JHEP 07, 115 (2011), 1105.4167.

[18] Y. Brihaye, C. Herdeiro, and E. Radu, Phys. Lett. B739, 1 (2014), 1408.5581.

[19] C. Herdeiro, J. Kunz, E. Radu, and B. Subagyo, Phys. Lett. B748, 30 (2015), 1505.02407.

[20] S. Ponglertsakul, E. Winstanley, and S. R. Dolan, Phys. Rev. D94, 024031 (2016), 1604.01132.

[21] S. Ponglertsakul and E. Winstanley (2016), 1610.00135.

[22] F. E. Schunck and E. W. Mielke, Class. Quant. Grav. 20, R301 (2003), 0801.0307.

[23] R. Brito, V. Cardoso, C. A. R. Herdeiro, and E. Radu, Phys. Lett. B752, 291 (2016), 1508.05395.

[24] F. Garca and I. S. Landea (2016), 1608.00011.

[25] M. Duarte and R. Brito, Phys. Rev. D94, 064055 (2016), 1609.01735 .

[26] D. Kastor and J. H. Traschen, Phys. Rev. D46, 5399 (1992), hep-th/9207070.
[27] C. A. R. Herdeiro and E. Radu, Int. J. Mod. Phys. D23, 1442014 (2014), 1405.3696.

[28] P. J. Montero and I. Cordero-Carrion, Phys.Rev. D85, 124037 (2012), 1204.5377.

[29] N. Sanchis-Gual, J. C. Degollado, P. J. Montero, and J. A. Font, Phys. Rev. D 91, 043005 (2015), 1412.8304.

[30] N. Sanchis-Gual, J. C. Degollado, P. J. Montero, J. A. Font, and V. Mewes, Phys. Rev. D 92, 083001 (2015), 1507.08437.

[31] I. Cordero-Carrión and P. Cerdá-Durán, ArXiv e-prints (2012), 1211.5930.

[32] I. Cordero-Carrión and P. Cerdá-Durán, Advances in Differential Equations and Applications, SEMA SIMAI Springer Series Vol. 4 (Springer International Publishing Switzerland, Switzerland, 2014).

[33] M. Alcubierre, Introduction to 3+1 Numerical Relativity (Oxford Univ. Press, New York, 2008), ISBN 978-0-19920567-7.

[34] R. Brito, V. Cardoso, and P. Pani, Lect. Notes Phys. 906, pp.1 (2015), 1501.06570.

[35] P. Bosch, S. R. Green, and L. Lehner, Phys. Rev. Lett. 116, 141102 (2016), 1601.01384.

[36] R. Li and J. Zhao, Eur. Phys. J. C74, 3051 (2014), 1403.7279.

[37] R. Li, J.-K. Zhao, and Y.-M. Zhang, Commun. Theor. Phys. 63, 569 (2015), 1404.6309.

[38] R. Li and J. Zhao, Phys. Lett. B740, 317 (2015), 1412.1527.

[39] R. Li, J. Zhao, X. Wu, and Y. Zhang, Eur. Phys. J. C75, 142 (2015), 1501.07358.

[40] I. Sakalli and G. Tokgoz, Adv. High Energy Phys. 2015, 739153 (2015), 1502.02274.

[41] R. Li, Y. Tian, H.-b. Zhang, and J. Zhao, Phys. Lett. B750, 520 (2015), 1506.04267.

[42] Y. Huang, D.-J. Liu, and X.-Z. Li (2016), 1606.00100.

[43] S. Hod, Phys. Lett. B761, 326 (2016).

[44] E. Seidel and W.-M. Suen, Phys. Rev. D42, 384 (1990).

[45] M. Alcubierre, F. S. Guzman, T. Matos, D. Nunez, L. A. Urena-Lopez, and P. Wiederhold, Class. Quant. Grav. 19, 5017 (2002), gr-qc/0110102.

[46] F. S. Guzman, Phys. Rev. D70, 044033 (2004), grqc/0407054.

[47] A. Bernal and F. S. Guzman, Phys. Rev. D74, 063504 (2006), astro-ph/0608523.

[48] A. Bernal, J. Barranco, D. Alic, and C. Palenzuela, Phys. Rev. D81, 044031 (2010), 0908.2435.

[49] I. Pena and D. Sudarsky, Class. Quant. Grav. 14, 3131 (1997). 